\section{A selected review of abstracts from the 10th Congress of the European Association of Neuro-Oncology}

Marc C Chamberlain*

10th European Association of Neuro-Oncology, Marseille, France, 6-9 September 2012

The European Association of Neuro-Oncology (EANO) is the largest neurooncology meeting in Europe. It meets biannually and reproducibly provides an exciting forum to present new brain cancer clinical trials and research data. The EANO 2012 meeting comprised 3 days of presentations; nearly 80 oral presentations and over 350 abstracts, providing a contemporary overview of neuro-oncology that includes both metastatic diseases of the CNS as well as primary brain tumors. This article attempts to highlight select abstracts presented at this year's meeting in a short review that provides a portrait of a large and multifaceted meeting.

\section{Metastatic disease to the CNS}

- Parenchymal brain metastases

Several abstracts were presented regarding brain metastasis. Most notable was a large retrospective series of patients $(\mathrm{n}=777)$ treated with surgery followed by wholebrain irradiation (WBI) or WBI alone [1]. Outcome was determined to be dependent upon recursive partitioning analysis class, graded prognostic assessment class, performance status and tumor histology. The best outcome was seen in breast cancer brain metastases. From the same institution, 329 patients with one or two brain metastases were retrospectively evaluated for outcome, comparing surgery followed by WBI and a stereotactic radiotherapy boost (SRB) versus WBI and, in responding patients, a $S R B$ [2]. The group treated by surgery followed by WBI and SRB resulted in the best outcome and breast cancer-derived metastases benefited most from WBI and SRB.

\section{- Leptomeningeal disease}

A study from Spain demonstrated the safety of intra-cerebrospinal fluid (CSF) chemotherapy as determined by the incidence of treatment-related side effects. Furthermore, the study noted that side effects were disproportionately higher in patients treated by a ventricular access device or in those patients receiving intra-CSF liposomal cytarabine [3]. Another study from Spain utilized CSF flow cytometry and a monoclonal antibody directed against epithelial cell adhesion molecule in patients with carcinomatous meningitis and determined a $75 \%$ detection rate of malignant cells in the CSF [4]. The authors postulate tumor burden, as determined by the proportion of positive cells, has survival implications.

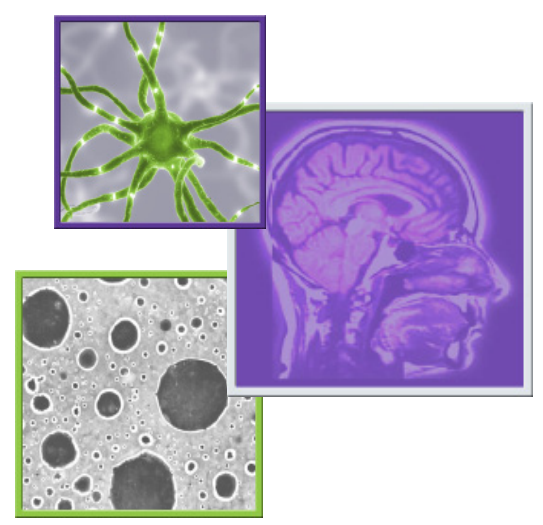

\section{News \& Views}

News

Journal Watch

Ask the Experts

Interview

Conference Scenes

*University of Washington, Department of Neurology/Division of Neuro-Oncology, Fred Hutchinson Cancer Research Center, Seattle Cancer Care Alliance, 825 Eastlake Avenue E, POB 19023, MS G4940, Seattle, WA 98109 1023, USA; Tel.: +1 206288 8280; Fax: +1 206288 2000; chambemc@u.washington.edu 
However, no comparison with CSF cytology was provided. A retrospective study of 240 patients with leptomeningeal disease (165 with solid tumors, 50 with lymphoma and 25 with leukemia) demonstrated that, following pretreatment neuraxis imaging (including brain and complete spine MRI) and radioisotope CSF flow study, approximately a third of patients had radiographic abnormalities that altered leptomeningeal disease-directed treatment [5].

\section{Primary brain tumors}

- Primary CNS lymphoma

The Bonn group treated 89 elderly patients with primary CNS lymphoma (PCNSL) in a prospective Phase II trial using a systemic polychemotherapy (which included highdose methotrexate and cytarabine) and a intra-CSF liposomal cytarabine regimen [6]. Although treatment-related mortality and morbidity were high (nearly $25 \%$ ), a third of patients survived for over 5 years. The International PCNSL Collaborative Group presented retrospective data on 48 patients with primary leptomeningeal CNS lymphoma [7]. The outcome did not appear to differ according to the treatment administered and median overall survival was 18 months. In a large study $(\mathrm{n}=249)$ of genomic alterations in patients with PCNSL, the five most frequent abnormalities were $6 \mathrm{p}$ loss (corresponding to HLA loss), 6q loss, $C D K N 2 A$ homozygous deletions, $12 \mathrm{q}$ loss and $7 \mathrm{q}$ gains [8]. CDKN2A homozygous deletions and $6 \mathrm{q}$ loss were associated with shorter survival.

\section{- Meningiomas}

A study from Germany assessed the methylation status of the TIMP-3 gene, which localizes to chromosome 22 and is frequently lost in meningioma. The study demonstrated that the TIMP-3 gene is often methylated, thereby silencing TIMP-3 expression and, presumably, facilitating tumor invasion. TIMP-3 promoter methylation was more frequently seen in high-grade meningiomas [9]. In a prospective two-step Phase II trial of patients with recurrent highgrade meningioma $(\mathrm{n}=7)$ using a long-acting somatostatin analog, the primary end point (radiographic response) was not met (no objective responses were seen). However, the median and 6-month progression-free survival were 4.2 months and $43 \%$, respectively, similar to a prior retrospective study of a long-acting somatostatin analog in recurrent meningioma [10].

\section{- Gliomas}

Low-grade gliomas

A provocative study from France suggested that neoadjuvant temozolomide (TMZ) chemotherapy can, in selected patients, reduce tumor volume, thereby permitting an extensive resection [11]. This was not considered feasible prior to TMZ. Two studies emphasized the importance of PET imaging (using differing radioligands, ${ }^{18}$ fluroethyl-tyrosine and ${ }^{11} \mathrm{C}$-methionine) in determining tumor grade and outcome in tumors that, by anatomic MRI, appear to be low-grade gliomas [12,13]. Both studies suggested that increased uptake by the amino acid radioligand predicted higher tumor grade and decreased survival. An Italian prospective Phase II trial of dosedense TMZ in patients with progressive or recurrent oligodendroglioma demonstrated a partial response rate of $35 \%$, a significant reduction (defined as a $>50 \%$ reduction of pretreatment frequency of seizures) in seizures (seen in $85 \%$ of patients) and a median progression-free survival of over 33 months [14]. Whether these results differ significantly from standard-dose TMZ is uncertain and perhaps warrants a prospective randomized trial, as suggested by the authors.

\section{Anaplastic gliomas \\ Upfront trials}

The long-term follow-up study to the European Organization for the Research and Treatment of Cancer (EORTC) trial for newly diagnosed anaplastic oligodendroglial tumors (AOT) was presented again and demonstrated a significant survival advantage for the codeleted 1p19q cohort of AOT treated with adjuvant radiotherapy plus procarbazine, lomustine and vincristine (PCV) chemotherapy [15]. This study, along with the companion Radiation Treatment Oncology Group (RTOG) study, is clinically relevant as both demonstrate a twofold increase in median overall survival in patients with codeleted 1p19q AOT treated initially with 
radiotherapy plus PCV compared with radiotherapy alone followed at recurrence with an alkylator-based chemotherapy (12-14 vs 7 years). Futhermore, there was no survival advantage seen in the larger group of patients with AOT who are uni- or nondeleted when comparing radiotherapy plus $\mathrm{PCV}$ with radiotherapy, suggesting a limited (if any) effect of adjuvant PCV on survival in patients with nondeleted anaplastic glioma. The potential benefit of adjuvant chemotherapy is being addressed in another prospective Phase III EORTC trial (CATNON), which is comparing radiotherapy with radiotherapy plus TMZ, and is open and actively accruing. In an analysis of data from the German NOA-04 trial (radiotherapy vs TMZ or PCV chemotherapy), methylation of the tumor $M G M T$ gene promoter was prognostic and associated with improved median overall survival in patients with an IDHI mutation whether patients received radiotherapy or TMZ/PCV [16]. However, in patients with wild-type $I D H 1$ and methylated $M G M T$, a survival advantage was only seen in patients who were administered TMZ/PCV.

\section{Glioblastoma \\ Upfront trials}

Two presentations outlined novel adjuvant immunotherapy approaches $[17,18]$. One discussed a Phase I trial that used a peptide vaccine derived from 11 HLA-binding tumor-specific antigens combined with standard-of-care radiotherapy plus TMZ. The preliminary work suggests that the vaccine was safe when combined with standard-of-care radiotherapy plus TMZ [17]. The other presentation discussed a preclinical study in which the immune response in mice to an IDH1 peptide vaccine derived from the common $\mathrm{R} 132 \mathrm{H}$ mutation was assessed and found to be immunogenic [18]. Both of these studies add to the increasing literature available regarding the applicability of immunotherapy in the management of glioblastoma (GBM). The German NOA-08 trial (elderly patients with GBM or anaplastic astrocytoma treated with standard-dose radiotherapy, $60 \mathrm{~Gy}$ or dose-dense TMZ) was again presented, demonstrating a survival advantage with dose-dense TMZ treatment in elderly patients with tumors showing promoter methylation of the $M G M T$ gene [19]. This, and a similar Nordic trial (elderly GBM patients treated with standard or hypofractionated radiotherapy vs standarddose TMZ), suggests that elderly patients with GBM show a similar survival benefit when treated with TMZ only and deferred radiotherapy when compared with radiotherapy only. In addition, survival is improved in the small cohort $(-30 \%)$ of elderly patients with GBM and MGMTmethylated tumors when treated with TMZ, a practice-changing treatment strategy. Two imaging studies assessed apparent progression following completion of radiotherapy plus TMZ in an attempt to differentiate between pseudoprogression and early disease progression [20,21]. In a French retrospective cohort of 58 patients, very early disease progression (progression while receiving radiotherapy plus TMZ) was seen in $20 \%$, pseudoprogression in $17 \%$ and early disease progression in $33 \%$ of patients [20]. Using a novel blood pool agent, ferumoxytol, which, unlike gadolinium, does not extravasate into the tumor interstitium, relative cerebral blood volume (rCBV) was determined with perfusion MRI using both contrast agents [21]. Ferumoxytol-determined rCBV was superior in differentiating pseudoprogression (defined as low rCBV) from early disease progression (high rCBV).

\section{Salvage trials}

Two studies correlated response to bevacizumab with advanced imaging [22,23]. A small French study suggested serial fluorodeoxyglucose-PET imaging, when performed at baseline and repeated while patients were receiving bevacizumab, predicts survival [22]. This study, however, conflicts with the National Cancer Institute (NCI) trial of bevacizumab for recurrent GBM and suggests, at present, that there is not a radiologic biomarker predictive of response to angiogenic inhibition. In a German retrospective study of 30 patients on bevacizumab, advanced MRI using susceptibility-weighted sequences demonstrated that hypodense susceptibility signals predicted for failure on bevacizumab [23]. The source of the susceptibility signal is not fully understood, 
although they hypothesized it to result from tumor invasion, co-option of cerebral vasculature and microbleeds.

\section{Quality of life}

Two studies evaluated quality of life (QoL) in caregivers of patients with gliomas $[24,25]$. Both studies demonstrate that caregivers of patients with brain tumors have a compromised QoL when compared with caregivers with non-CNS cancers. In part, these differences reflect the unique care situation in patients with brain tumors as disease progression, mobility and cognition are negatively affected. Another study in patients with gliomas suggests a marked discordance between patients and their caregivers when reporting QoL; patients appear to consistently rank QoL higher than their caregivers throughout the disease course [26]. This finding emphasizes the importance of including the caregiver assessment of patient QoL. The EORTC trial of AOT alluded to above assessed cognition and QoL in a small cohort of patients

\section{References}

1 Antoni D, Clavier J, Noel G. A retrospective analysis of 777 patients with brain metastases: overall survival and prognostic factors. Neuro Oncol. 14(Suppl. 13), iii77 (2012) (Abstract P.236).

2 Antoni D, Clavier J, Noel G. Role of surgery and postoperative radiotherapy for 329 patients RPA I or II with 1 or 2 brain metastases. Neuro Oncol. 14(Suppl. 13), iii77 (2012) (Abstract P.237).

3 Pardo J, Cuadrado MI, Fernnandez C. Safety of intrathecal themotherapy: an analysis of 627 procedures. Neuro Oncol. 14(Suppl. 13), iii78 (2012) (Abstract P.238).

4 Subira D, Illan J, Serrano C et al. Prognostic impact of flow cytometry quantification of $\mathrm{EPCAM}^{+}$cells in the cerebrospinal fluid of patients with leptomeningeal cancinomatosis. Neuro Oncol. 14(Suppl. 13), iii75 (2012) (Abstract P.227).

5 Chamberlain MC. Neuraxis imaging in leptomeningeal metastasis: a retrospective case series. Neuro Oncol. 14(Suppl. 13), iii74 (2012) (Abstract P.223).

6 Kuhnhenn J, Pels H, Reiser $\mathrm{M}$ et al. Combined systemic polychemotherapy and intrathecal treatment with liposomal cytarabine in patients with primary CNS lymphoma $\geq 60$ years: results of Phase IITrial. Neuro Oncol. 14(Suppl. 13), iii (2012) (Abstract O.32).

7 Schiff D, Taylor JW, Flanagan E et al. Presentation, diagnostics and treatment of primary leptomeningeal lymphoma: an international primary CNS lymphoma collaborative group series. Neuro Oncol. 14(Suppl. 13), iii9 (2012) (Abstract O.33).

8 Gonzalez-Aguilar A, Boisselier B, Polivka M et al. Recurrent genomic alterations in primary CNS lymphoma. Neuro Oncol. 14(Suppl. 13), iii84 (2012) (Abstract P.261).

9 Ketter R, Linsler S, Kramer D et al. Hypermethylation of TIIMP3 is a predictive diagnostic marker in meningiomas. Neuro Oncol. 14(Suppl. 13), iii70 (2012) (Abstract P.210).

10 Simo M, Macia M, Plans G et al. Recurrent high-grade meningioma: Phase II trial with somatostatin analogue therapy. Neuro Oncol. 14(Suppl. 13), iii8 (2012) (Abstract O.30).

11 Blonski M, Pallud J, Goze C et al. Regardless of the molecular status, neoadjuvant chemotherapy can optimize the extent of resection of World Health Organization grade II gliiomas. Neuro Oncol. 14(Suppl. 13), iii65 (2012) (Abstract P.187). $(\mathrm{n}=35)$ compared with controls 2.5 years after treatment [27]. Outcome was variable, wherein $41 \%$ of patients did not show significant cognitive impairment, $25 \%$ were mildly to moderately impaired, $31 \%$ were severely impaired and $78 \%$ were independent in activities of daily living. Both recurrent seizures and disease progression negatively affected cognitive performance; however, there appeared to be no difference in outcome with regard to primary therapy (radiotherapy or radiotherapy plus PCV).

\section{Financial \& competing interests disclosure}

The author has no relevant affiliations or financial involvement with any organization or entity with a financial interest in or financial conflict with the subject matter or materials discussed in the manuscript. This includes employment, consultancies, honoraria, stock ownership or options, expert testimony, grants or patents received or pending, or royalties.

No writing assistance was utilized in the production of this manuscript.

12 Kunz M, Armbruster L, Thon $\mathrm{N}$ et al. Hot-spots in dynamic FET PET are associated with unfavorable outcome in patients with suspected WHO grade II glioma. Neuro Oncol. 14(Suppl. 13), iii (2012) (Abstract O.40).

13 Berntsson S, Savitcheva I, Larsson E, Smits A. ${ }^{11} \mathrm{C}$-methionine PET combined with advanced MRI for the preoperative evaluation of suspected diffuse low-grade gliomas. Neuro Oncol. 14(Suppl. 13), iii11 (2012) (Abstract O.41).

14 Ruda R, Bertero L, Trevisan E et al. Dose-dense temozolomide as initial treatment for progressive low grade oligodendroglial tumors: a multicenter Phase II study of the AINO (Italian Association of Neuro-Oncology). Neuro Oncol. 14(Suppl. 13), iii11 (2012) (Abstract O.39).

15 van den Bent MJ, Brandes AA, Taphoorn JB et al. Long term follow-up results of EORTC 26951: a randomized Phase III study on adjuvant PCV chemotherapy in an anaplastic oligodendroglial tumors - a report of the EORTC Brain Tumor Group. Neuro Oncol. 14(Suppl. 13), iii12 (2012) (Abstract O.42).

16 Wick W, Meisner C, Hentschel B et al. Prognostic or predictive value of MGMT 
promoter methylation malignant gliomas depends on IDH1 mutations. Neuro Oncol. 14(Suppl. 13), iii7 (2012) (Abstract O.25).

17 Rampling RP, James A, Mulholland P et al. A first in man Phase I trial of IMA950 (a novel multi-peptide vaccine) plus GM-CSF in patients with newly diagnosed glioblastoma - design and preliminary results of Cancer Research UK study. Neuro Oncol. 14(Suppl. 13), iii6 (2012) (Abstract O.22).

Schumacher T, Sahm F, von Deimling A,

Wick W, Platten M. IDH1 mutation: immunotherapy for malignant glioma? Neuro Oncol. 14(Suppl. 13), iii6 (2012) (Abstract O.23).

19 Weller M, Meisner C, Platten M et al. MGMT promoter methylation predicts benefit from temozolomide versus radiotherapy in malignant astrocytomas in the elderly: the NOA-08 trial. Neuro Oncol. 14(Suppl. 13), iii12 (2012) (Abstract O.43).
20 Wertz M, Padovani L, Bequet-Boucard C et al. Is pseudoprogression (PSP) impacted by a very early progression (VEP)? A retrospective cohort study. Neuro Oncol. 14(Suppl. 13), iii57 (2012) (Abstract P.156).

21 Gahramanov S, Muldoon LL, Varallyay CG et al. Radiographic diagnosis of pseudoprogression and correlation with survival of patients with glioblastoma after chemoradiotherapy using dynamic susceptibility-weighted contrast-enhanced perfusion MR imaging with ferumoxytol vs. gadotheridol. Neuro Oncol. 14(Suppl. 13), iii36 (2012) (Abstract P.077).

22 Colavolpe C, Chinot O, Metellus P et al. FDG-PET predicts survival in recurrent high grade gliiomas treated with bevacizumab and irinotecan. Neuro Oncol. 14(Suppl. 13), iii36 (2012) (Abstract O.36).

23 Radbruch A, Kramp L, Wiestler B, Heiland S, Wick W, Bendszus M. Susceptibility weighted imaging predicts progressive disease in patients with recurrent glioblastoma treated with bevacizumab. Neuro Oncol. 14(Suppl. 13), iii10 (2012) (Abstract O.35).

24 Boele FW, Jeimans JJ, Aaronson NK et al. Health-related quality of life in spouses of glioma patients compared with spouses of patients with non-CNS tumors. Neuro Oncol. 14(Suppl. 13), iii4 (2012) (Abstract O.15).

25 Flores PM, Chinot O, Berbis J, Auquier P. Assessing the impact of brain cancer in caregivers' quality of life (QOL). Neuro Oncol. 14(Suppl. 13), iii5 (2012) (Abstract O.17).

26 Kumthekar PU, Grimm SA, Chandler J et al. Quality of life concording between patients with malignant bliomas (MG) and their caregivers: final data. Neuro Oncol. 14(Suppl. 13), iii5 (2012) (Abstract O.18).

27 Habets EJJ, Taphoorn JB, Nederend S et al. Cognitive functioning and health related quality of life in long-term anaplastic glioma survivors from EORTC study. Neuro Oncol. 14(Suppl. 13), iii4 (2012) (Abstract O.16). 